\title{
The industrialization of ablation: a highly standardized and reproducible workflow for radiofrequency ablation of atrial fibrillation
}

\author{
Tom De Potter ${ }^{1}$ (1) - Tina D. Hunter ${ }^{2}$ - Lee Ming Boo ${ }^{3} \cdot$ Sofia Chatzikyriakou ${ }^{1,4}$. \\ Teresa Strisciuglio $^{1,5} \cdot$ Etel Silva $^{1} \cdot$ Peter Geelen $^{1}$
}

Received: 14 March 2019 / Accepted: 6 September 2019 / Published online: 17 October 2019

(C) The Author(s) 2019

\begin{abstract}
Background or Purpose The purpose of this analysis was to report on efficacy of a standardized workflow for atrial fibrillation (AF) ablation using technology advances such as 3D imaging and contact force sensing in a real-world setting.

Methods Consecutive AF ablations from 2014 to 2015 at a high-volume site in Belgium were included. The workflow consisted of a pre-specified procedure sequence including 3D modeling followed by radiofrequency encircling of the pulmonary veins (25 W posterior wall, $35 \mathrm{~W}$ anterior wall) with a THERMOCOOL SMARTTOUCH® Catheter guided by CARTO VISITAGTM Module $(2.5 \mathrm{~mm} / 5 \mathrm{~s}$ stability, $50 \%>7 \mathrm{~g}$ ) and ablation index (targets: 550 anterior wall, 400 posterior wall). Efficiency endpoints were procedure time, fluoroscopy time, and radiation dose. The primary effectiveness endpoint was freedom from atrial arrhythmia recurrence.

Results A total of 605 paroxysmal AF (PAF) and 182 persistent AF (PsAF) patients were followed for $436 \pm 199$ days. Mean procedure times were short (PAF: $96.1 \pm 26.2 \mathrm{~min}$; PsAF: $109.2 \pm 35.6 \mathrm{~min})$ with most procedures $(90.6 \%$ PAF; $81.3 \%$ PsAF) completed in $\leq$ $120 \mathrm{~min}$. Minimal fluoroscopy was utilized (PAF: $6.1 \pm 3.8 \mathrm{~min}, 5.9 \pm 3.4 \mathrm{~Gy}^{*} \mathrm{~cm}^{2}$; PsAF: $6.9 \pm 4.7$ min, $7.4 \pm 4.9$ Gy* $\mathrm{cm}^{2}$ ). Freedom from atrial arrhythmia recurrence was higher for PAF than PsAF patients (OR: 2.0, 95\% CI: 1.4-2.9, $p=0.0003$ ), but adjusted mean rates were high in both groups (81.0\% vs. 67.9\%). Rates were adjusted for prior ablation and age (at 65 years).

Conclusion AF ablation using a standardized workflow resulted in low procedure times and variability, with minimal fluoroscopy exposure. Long-term freedom from atrial arrhythmia recurrence was high in both PAF and PsAF populations.
\end{abstract}

Keywords Atrial fibrillation · Catheter ablation · Arrhythmias

\section{Introduction}

Real-time contact force (CF)-sensing catheters have been shown to improve ablation outcomes when compared to non-CF technologies in atrial fibrillation (AF) ablation [1].

Tom De Potter

tom.de.potter@olvz-aalst.be

1 Cardiovascular Center, OLV Hospital, Moorselbaan 169, 9300 Aalst, Belgium

2 CTI Clinical Trial and Consulting Services, Covington, KY, USA

3 Biosense Webster, Inc, Irvine, CA, USA

4 Cardiology Department, CUB-Hôpital Erasme, Université Libre de Bruxelles, Brussels, Belgium

5 University of Naples Federico II, Naples, Italy
Ablation Index (AI), which integrates CF, power, and ablation time in a logarithmic formula, was shown to be an independent predictor of pulmonary vein (PV) reconnection [2]. Further, a recent study in 100 patients showed that a standardized AI-guided workflow improved 1-year outcomes compared to non-standardized CF ablation [3]. Use of these combined technologies allows for standardization of PV ablation workflow, leading to increased predictability.

The objective of this study was to assess whether the use of a highly standardized workflow, combining CF-sensing technology with AI and visualization of lesion durability, positively impacts the procedural efficiency and effectiveness of radiofrequency $(\mathrm{RF})$ ablation in large real-world paroxysmal AF (PAF) and persistent AF (PsAF) populations. This study reports on improvements in the levels and variability of procedural efficiency measures - procedural duration and fluoroscopy use in particular, as well as freedom from atrial arrhythmia recurrence rates through the 12-month visit. 


\section{Methods}

This retrospective cohort study analyzed outcomes of consecutive AF ablations from a high-volume cardiovascular center in Belgium between January 2014 and December 2015. Subjects underwent RF ablation using the THERMOCOOL SMARTTOUCH ${ }^{\circledR}$ Catheter (ST; Biosense Webster, Inc.) guided by CARTO VISITAG ${ }^{\mathrm{TM}}$ Module (Visitag; Biosense Webster, Inc.) with AI. Baseline patient characteristics, procedural efficiency, and effectiveness outcomes were collected for all ablations during the study period and analyzed according to AF type (PAF vs. PsAF).

All patients gave written consent for the collection and analysis of their data. The Medical Ethics Committee of the OLV Hospital approved the use of the registry data for this study on July 13, 2016. All data used to perform the statistical analyses were de-identified and accessed in compliance with the Health Insurance Portability and Accountability Act.

\subsection{Population}

Study subjects were consecutive adult patients who presented for an AF catheter ablation using an RF approach at the study site during the 2-year period. Subjects were evaluated as candidates for the procedure according to standard clinical practices with no additional criteria for study inclusion or exclusion.

\subsection{Workflow for ablations}

All procedures used a 3D anatomic model generated from real-time 3D rotational angiography (3DRA) integrated automatically in the mapping system, with no additional catheterbased geometry reconstruction, using a workflow described previously and summarized below [4].

\subsubsection{D image acquisition and integration}

A $6 \mathrm{~F}$ pigtail catheter was positioned in the center of the LA after transseptal puncture, with the pigtail positioned in the isocenter of the fluoroscopy system (GE Innova 2100) using $\mathrm{AP}$ and lateral projections. Prior to acquisition, the imaging system was registered to the CARTO system using the Univu module. During fast ventricular pacing (220-240 ms), $100 \mathrm{cc}$ of contrast medium (Ultravist, Schering, Germany) was injected in the LA over $5 \mathrm{~s}$. The $\mathrm{C}$-arm was rotated around the patient during $4.5 \mathrm{~s}$ in an automatic sequence, acquiring near-simultaneous 2D frames during apnea. At the end of the 3DRA acquisition, the dataset was automatically transported to an imaging workstation and a 3D model was constructed using a cone beam reconstruction algorithm (Advance Workstation 3, GE). This 3D model was then transferred over the network to the CARTO workstation for integration with the electroanatomical coordinate system. Because acquisition of the 3DRA was performed with the Univu image integration functionality active, the individual background frames of the 3DRA were visible in the CARTO system in the appropriate spatial positions, allowing them to be used to visually align the 3D model with those frames. Thus, an electroanatomical shell was not required for registration, as is the case in conventional CARTOMERGE workflows. A schematic of this workflow is provided in Fig. 1.

\subsubsection{Standardized ablation workflow}

Pulmonary veins were isolated by sequential RF encircling with the ST catheter using this 3D model. Ipsilateral veins were ablated in pairs using a wide area circumferential ablation approach (WACA). Power settings were $25 \mathrm{~W}$ at the posterior wall and $35 \mathrm{~W}$ at the anterior wall, using recommended labeling-indicated flow rates. Standardized Visitag settings (maximum location stability range: $2.5 \mathrm{~mm}$, minimum time: $5 \mathrm{~s}$, and minimum force over time: $50 \%>7 \mathrm{~g}$ ) and AI settings (targeting 550 at the anterior wall and 400 at the posterior wall) were used with a maximum interlesion distance target of $8 \mathrm{~mm}$.

In all de novo ablation patients, ablation was restricted to pulmonary vein isolation (PVI), regardless of AF type, except for patients with documented typical right atrial flutter for whom an additional cavotricuspid isthmus ablation was performed. For re-ablations, repeat PVI was performed by targeted ablation on the previously created WACA line, guided by activation patterns, and additional linear and focal ablations were performed as deemed necessary by the operator. In the case of linear lesions, bidirectional block across the line(s) was verified after ablation.

\subsection{Data collection and follow-up}

Baseline patient characteristics, including pre-procedure test results, treatment history, comorbid conditions, and stroke risk scores, were recorded pre-ablation. Efficiency measures of procedure time, fluoroscopy time, and radiation dose were captured. Patients were monitored for procedure-related complications including tamponade and/or pericardial effusion, thromboembolic events, and bleeding events, but structured information was only collected prospectively and systematically beginning in 2015 . Prior to 2015 , only complications that led to clinical events such as death, stroke, or tamponade were collected. Beginning in 2015, active collection of subclinical events such as a hematoma was added, even when they did not lead to a new medical contact.

Follow-up visits typically occurred at 3, 6, and 12 months post-ablation, at which time patients were monitored for atrial arrhythmia recurrence via questionnaire and ECG recording, with a 24-h Holter monitor at the end of follow-up. Atrial 

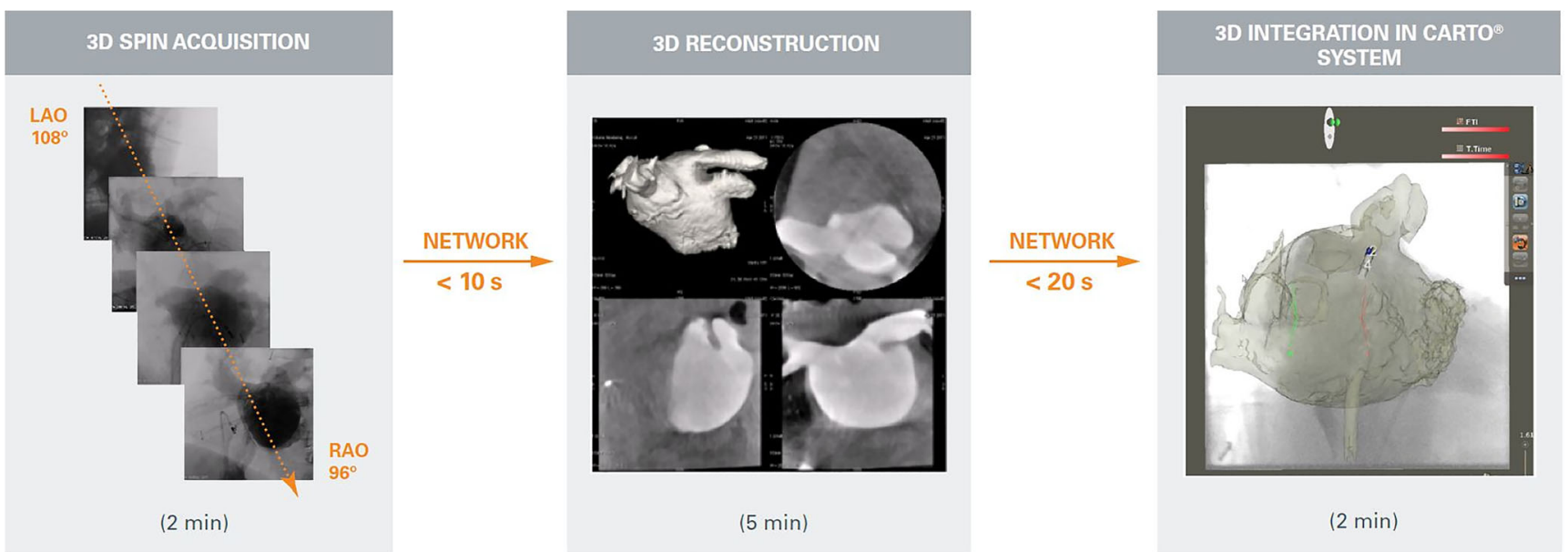

Fig. 1 Ablation workflow schematic. *Segmentation could also be done with CARTO® "image integration" tool. In that case, the output of the 3D reconstruction can be directly retrieved from CARTO ${ }^{\circledR}$

arrhythmia recurrence included AF, atrial tachycardia, or atrial flutter. Arrhythmia events occurring in the first month after ablation were blanked from the analysis and no repeat ablations were performed within the first 3 months. Additional unscheduled Holter monitoring was performed in cases of undocumented / unexplained symptoms. Antiarrhythmic drugs (AAD), oral anticoagulation status, and AF symptoms were also recorded.

\subsection{Statistical analysis}

Baseline patient characteristics, procedural efficiency measures, complications, and 12-month effectiveness outcomes were summarized by AF type. Multivariable logistic regression was used to model freedom from atrial arrhythmia recurrence through the latest follow-up visit. The explanatory variables of primary interest were baseline AF type (PAF or PsAF) and de novo ablation vs. re-ablation. Covariates that were tested for statistical significance included length of follow-up and baseline patient characteristics. Only variables that were statistically significant at a level of $\alpha=0.10$ were retained in the final model.

All statistical analyses in this study were performed using SAS software, Version 9.2 or higher (SAS Institute, Inc., Cary, NC, USA).

\section{Results}

A total of 787 patients underwent catheter ablation for $\mathrm{AF}$ (605 PAF, 182 PsAF), and approximately half of these procedures were re-ablations (PAF: $46.8 \%$, PsAF: $47.3 \%$ ). There was a higher percentage of males in the PsAF group than in the PAF group (76.4\% vs. $65.6 \%$ ), and the PsAF patients were slightly older, at $65.4 \pm 9.8$ years vs. $62.7 \pm 11.1$ years (Table 1). Hypertension was the most prevalent comorbidity among both groups (PAF: 46.6\%, PsAF: 52.8\%) followed by mitral insufficiency (PAF: $31.1 \%$, PsAF: 42.9\%), which was primarily grade 1 . Baseline patient characteristics are summarized in Table 1.

\subsection{Procedural detail}

Procedural efficiency measures are shown in Table 2. Mean procedure times, which included $20 \mathrm{~min}$ of waiting time postPVI, were short (PAF: $96.1 \pm 26.2 \mathrm{~min}$; PsAF: $109.2 \pm$ $35.6 \mathrm{~min}$ ). The majority of procedures were performed in 120 min or less (PAF: 90.6\%; PsAF: 81.3\%). Mean fluoroscopy times were minimal in both the PAF $(6.1 \pm 3.8 \mathrm{~min})$ and PsAF groups $(6.9 \pm 4.7 \mathrm{~min})$, with corresponding radiation doses of $5.9 \pm 3.4 \mathrm{~Gy}^{*} \mathrm{~cm}^{2}$ and $7.4 \pm 4.9 \mathrm{~Gy}^{*} \mathrm{~cm}^{2}$, respectively. Acute PVI was achieved in all patients.

Though the collection of detailed components of the total procedure time was not performed as a part of this study for all patients, a separate study collected this detail on a subset of 20 patients from the PAF cohort. A summary of the times for each step through the conclusion of the initial PV isolation is summarized in Table 3 as a point of reference for the reader.

Serious procedure-related complications were infrequent, occurring in only $1.8 \%$ (8/435) of the patients with ablations in 2015, after the structured reporting of these events began (Table 2). These consisted of seven pericardial effusion (four requiring pericardiocentesis) and one bleeding complication. There were no stroke or cardiac tamponade events, and no deaths.

\subsection{Effectiveness}

Rates of freedom from atrial arrhythmia recurrence through the latest follow-up visit were significantly higher for PAF patients than for PsAF patients (OR: 2.0, 95\% CI: 1.4-2.9, $p=0.0003$, Table 4). Adjusted rate estimates were $81.0 \pm 1.6 \%$ for PAF vs. 
Table 1 Baseline patient characteristics

\begin{tabular}{|c|c|c|}
\hline & $\begin{array}{l}\text { PAF } \\
(N=605)\end{array}$ & $\begin{array}{l}\text { PsAF } \\
(N=182)\end{array}$ \\
\hline Age, years & $62.7 \pm 11.1$ & $65.4 \pm 9.8$ \\
\hline Less than 65 & $309(51.1)$ & $76(41.8)$ \\
\hline $65-74$ & $219(36.2)$ & $74(40.7)$ \\
\hline 75 and older & $77(12.7)$ & $32(17.6)$ \\
\hline Male & $397(65.6)$ & $139(76.4)$ \\
\hline Body mass index $\mathrm{kg} / \mathrm{m}^{2}$ & $27.1 \pm 4.2$ & $28.3 \pm 4.5$ \\
\hline Current smoker & $46(7.6)$ & $27(14.8)$ \\
\hline \multicolumn{3}{|l|}{ Patient medical history } \\
\hline Congestive heart failure & $38(6.3)$ & $35(19.2)$ \\
\hline Hypertension & $282(46.6)$ & $96(52.8)$ \\
\hline Diabetes & $68(11.2)$ & $27(14.8)$ \\
\hline Cerebrovascular accident & $43(7.1)$ & $10(5.5)$ \\
\hline End stage renal disease & $3(0.5)$ & $2(1.1)$ \\
\hline \multicolumn{3}{|l|}{$\mathrm{CHA}_{2} \mathrm{DS}_{2}$-VASc Score } \\
\hline $0-1$ & $283(46.8)$ & $79(43.4)$ \\
\hline$\geq 2$ & $306(50.6)$ & $99(54.4)$ \\
\hline Missing & $16(2.6)$ & $4(2.2)$ \\
\hline \multicolumn{3}{|l|}{ Baseline medications } \\
\hline Antiarrhythmic drugs & $499(82.5)$ & $151(83.0)$ \\
\hline Anticoagulation & $272(45.0)$ & $96(52.8)$ \\
\hline Ejection fraction $(\%)$ & $58.4 \pm 7.3$ & $53.9 \pm 9.6$ \\
\hline Collected for 2015 Procedures Only & $(N=327)$ & $(N=101)$ \\
\hline \multicolumn{3}{|l|}{ Mitral insufficiency } \\
\hline Grade 1 & $158(26.1)$ & $56(30.8)$ \\
\hline Grade 2 & $30(5.0)$ & $19(10.4)$ \\
\hline Grade 3 & $0(0.0)$ & $2(1.1)$ \\
\hline Grade 4 & $0(0.0)$ & $1(0.6)$ \\
\hline Left atrial size (ml) & $158.9 \pm 31.7$ & $194.4 \pm 42.6$ \\
\hline
\end{tabular}

Results displayed as $n(\%)$ or mean $\pm \mathrm{SD}$

$P A F$ : paroxysmal atrial fibrillation, $P S A F$ : persistent atrial fibrillation

$67.9 \pm 3.5 \%$ for PsAF (Fig. 2). Patients having de novo ablations had higher rates of freedom from recurrence versus those with prior ablations (OR: 1.9, 95\% CI: 1.3-2.7 $p=0.0004,80.5 \pm$ $2.2 \%$ vs. $68.6 \pm 2.7 \%$ ). The only additional statistically significant predictor of freedom from recurrence was lower age ( $p=$ 0.0410 ), which was standardized to 65 years for calculating the adjusted mean recurrence rates.

In the subset of 418 patients (322 PAF, 96 PsAF) with de novo ablations, first procedure freedom from recurrence rate estimates were higher in both cohorts than those seen in the respective full population cohort (PAF:85.7 $\pm 2.0 \%$, PsAF: $74.0 \pm 4.5 \%$, Fig. 2).

Re-ablation rates were $9.6 \%$ and $9.9 \%$ over mean followup times of $438 \pm 201$ days and $430 \pm 190$ days in the PAF and PsAF cohorts, respectively. AAD utilization was ongoing in
Table 2 Procedural detail

$\operatorname{PAF}(N=605) \quad \operatorname{PsAF}(N=182)$

\begin{tabular}{lll}
\hline Ablations performed & & \\
PVI only & $539(89.1)$ & $155(85.2)$ \\
PVI plus additional ablation lines & $63(10.5)$ & $27(14.8)$ \\
Missing & $3(0.5)$ & $0(0.0)$ \\
Acute pulmonary vein isolation & $605(100.0)$ & $182(100.0)$ \\
Total procedure time (minutes) & & \\
Mean \pm SD & $96.1 \pm 26.2$ & $109.2 \pm 35.6$ \\
Interquartile range (Q1, Q3) & $(80,110)$ & $(90,120)$ \\
Total fluoroscopy time (minutes) & & \\
Mean \pm SD & $6.1 \pm 3.8$ & $6.9 \pm 4.7$ \\
Interquartile range (Q1, Q3) & $(3.6,7.6)$ & $(3.7,8.5)$ \\
Radiation dose (Gy*cm $\left.{ }^{2}\right)$ & & \\
Mean \pm SD & $5.9 \pm 3.4$ & $7.4 \pm 4.9$ \\
Interquartile range (Q1, Q3) & $(4.0,6.5)$ & $(4.4,8.4)$ \\
Complications (captured in 2014 and 2015$)$ & \\
Stroke & $0(0.0)$ & $0(0.0)$ \\
Cardiac tamponade & $0(0.0)$ & $0(0.0)$ \\
Death & $0(0.0)$ & $0(0.0)$ \\
Complications (captured in 2015 only) & $(N=333)$ & $(N=102)$ \\
Pericardial effusion & $7(2.1)$ & $0(0.0)$ \\
Bleeding complication & $1(0.3)$ & $0(0.0)$ \\
\hline
\end{tabular}

Results displayed as $n(\%)$ unless otherwise noted

$P A F$ paroxysmal atrial fibrillation, $P S A F$ persistent atrial fibrillation, $P V I$ pulmonary vein isolation

$34.7 \%$ and $40.1 \%$ of the PAF and PsAF cohorts as of their last follow-up visit.

\section{Discussion}

The current analysis suggests that using a standardized AIguided workflow and integrated 3D angiography-derived models enables predictable procedural efficiency. Procedure

Table 3 Typical times for components of ablation procedure (subset of $N=20$ PAF ablations)

\begin{tabular}{ll}
\hline Component of procedure & Time (minutes) \\
\hline Groin puncture to 1st transseptal puncture & $4.5 \pm 0.9$ \\
1st transseptal puncture to pigtail insertion & $1.7 \pm 0.7$ \\
Pigtail insertion to end of 3DRA acquisition & $3.3 \pm 0.7$ \\
End of 3DRA acquisition to 2nd transseptal puncture & $3.2 \pm 1.2$ \\
2nd transseptal puncture to 1st RF application (LPV) & $9.9 \pm 1.2$ \\
1st RF application (LPV) to end of LPV ablation & $14.7 \pm 1.6$ \\
End of LPV ablation to end of RPV ablation & $15.0 \pm 2.3$
\end{tabular}

3DRA: 3D rotational angiography, $L P V$ : left pulmonary vein, $R P V$ : right pulmonary vein 
Table 4 Multivariable logistic regression models of freedom from atrial arrhythmia recurrence

\begin{tabular}{lll}
\hline Variable & $P$ value & $\begin{array}{l}\text { Odds ratio } \\
\text { (95\% Wald confidence interval) }\end{array}$ \\
\hline $\begin{array}{lll}\text { Full population: } \\
\quad \text { Paroxysmal AF }\end{array}$ & 0.0003 & $2.02(1.38,2.95)$ \\
De novo AF ablation & 0.0004 & $1.88(1.33,2.67)$ \\
Age (per year) & 0.0410 & $0.98(0.97,1.00)$ \\
De novo ablations only: & & \\
Paroxysmal AF & 0.0080 & $2.11(1.22,3.67)$ \\
\hline
\end{tabular}

$A F$ : atrial fibrillation

and fluoroscopy times under the standardized workflow were markedly shorter and less variable when compared to prior studies of CF-sensing technology $[1,5]$. For example, comparing the PAF cohort in our study to the published SMARTAF cohort, a $>2$-fold average procedure time reduction was observed (96 min vs. $222 \mathrm{~min}$ ) [5]. Perhaps even more important from a standardization perspective is the observed reduction in the variability of procedure times in a real-world setting of consecutive cases (standard deviation of procedure time: $26 \mathrm{~min}$ vs. $84 \mathrm{~min}$ in SMART-AF) [5].

Serious procedure-related complications were infrequent and within previously reported ranges [5], indicating that efficiency and effectiveness gains did not compromise patient safety. Freedom from atrial arrhythmia recurrence among PAF patients was higher than typical of previously published studies on CF-sensing technology [5]. Although procedural efficiency and effectiveness were greater in the PAF cohort compared to the PsAF cohort, the PsAF cohort also demonstrated good efficiency and long-term effectiveness, consistent with previous reporting of $\mathrm{CF}$ ablation compared to pre-CF ablation technology [6].

Despite improvements in clinical outcomes associated with the use of CF-sensing catheters and integrated electroanatomic mapping technologies [1,7], procedure and fluoroscopy times can still be lengthy, and radiation exposure remains a concern for both patients and healthcare professionals [8]. Prior to the AIfacilitated standardized workflow, procedure times at the study site varied widely from case to case and were generally between 90 and $120 \mathrm{~min}$ on the low end and $4-5 \mathrm{~h}$ on the high end. The improved efficiency, reflected in the predictably shorter procedure and fluoroscopy times seen in this analysis of AI-guided ablation, underscores the importance of recent advancements in $\mathrm{CF}$ technology that enable standardization of PVI workflow. The shorter and less variable procedure times could potentially translate to improved procedure scheduling and long-term cost savings [9]. Another strategy for shortening procedure time that has garnered significant attention over the past year is ablation with high power and short duration (HPSD), defined as 40-50 W with current catheters or up to $90 \mathrm{~W}$ with novel catheters that are currently in development [10-15]. If proven, HPSD ablation could further reduce the procedure time via a reduction in the time required for RF application.

In the presented workflow, real-time 3D modeling was derived from rotational angiography and the automatic integration of this modeling in the 3D mapping system allowed for drastic reduction in fluoroscopy use, all but eliminating the need for fluoroscopy during the ablation phase. When using a 3D mapping system, there was previously no way to position an external 3D model (e.g., from CT or MRI) within the coordinate system of the mapping model. This limitation has been overcome by creating a catheter-based $3 \mathrm{D}$ model, then registering the external model with landmarks and surface fitting. Importantly, our workflow does not require any additional mapping because the
Fig. 2 Adjusted mean rates of freedom from atrial arrhythmia recurrence. Error bars represent the standard error of the adjusted mean. Rates were calculated at 65 years of age. PAF: paroxysmal atrial fibrillation, PsAF: persistent atrial fibrillation

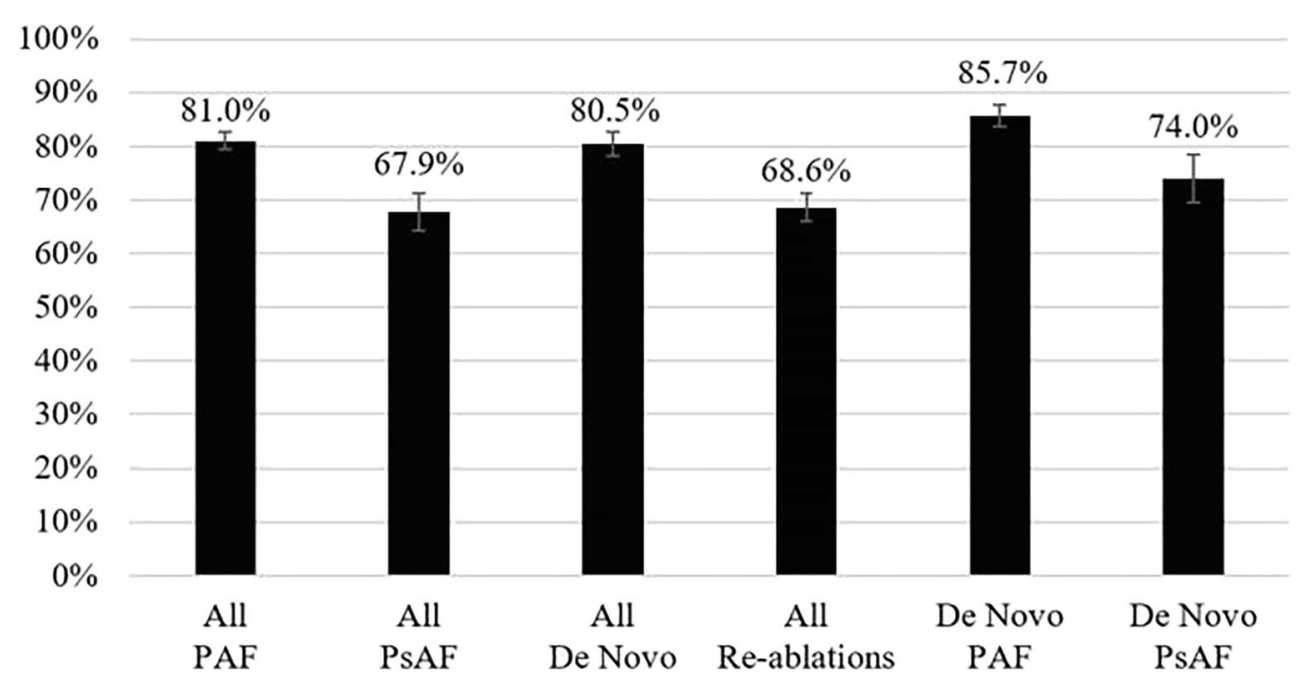

Population 
coordinates of the 3DRA model are linked to the CARTO system through the Univu module. Therefore, the time and fluoroscopy associated with $3 \mathrm{D}$ geometry creation, which varies depending on anatomy and operator experience, is eliminated. All steps required for the 3DRA processing, other than the acquisition (1 min total), are performed by a technician in parallel with ongoing steps of the procedure such that they do not impact overall procedure time.

If operators work with only the 3D model generated by the mapping system, the advantages of the workflow described above will obviously not apply, but there will be a different set of limitations. On top of the time and experience needed for acquisition of the $3 \mathrm{D}$ model by catheter manipulation, there is almost always a need for manual adjustment of these models (i.e., erasing certain regions, smoothening, adjusting mapping resolution) in order to obtain a high quality $3 \mathrm{D}$ representation. We believe that the 3DRA workflow presented here offers the best of both worlds by allowing a highly detailed 3D model to be acquired and integrated in the mapping system without manipulation, adjustment or fine-tuning.

The presented numbers reflect the fluoroscopy needed for procedural set-up (catheter placement, transseptal puncture) and 3D acquisition. Fluoroscopy exposure was primarily driven by the 3D angiography acquisition, during which the operator was outside of the room and not exposed to radiation. Therefore, typical operator exposure doses were well below 1 microSievert during the PVI procedure per real-time electronic dosimeter recordings (data not presented here). Alternatively, similarly low exposure rates could be achieved with the use of intracardiac echocardiography. However, this modality requires time and operator experience and is associated with prohibitively high costs in most healthcare reimbursement models outside the US.

Our results demonstrate that both PAF and PsAF patients had lower recurrence rates than those commonly seen among comparable groups receiving $\mathrm{RF}$ ablation [5, 16, 17]. A recent study from a single center using a similar standardized AI workflow showed similar results with an overall single procedure success rate of $>90 \%$ in PAF patients [3, 18]. In addition to an AI target value, the aforementioned study also utilized an interlesion distance of $\leq 6 \mathrm{~mm}$ to ensure lesion contiguity around the PV. Our study used a target interlesion distance of $\leq 8 \mathrm{~mm}$ based on an earlier retrospective analysis of our site data.

Given that PVI is the cornerstone for all AF ablation, it is possible that AI facilitates stable catheter-tissue contact for consistent and more durable lesion creation around the PVs, thereby improving subsequent long-term outcomes. The associations of catheter stability and lesion contiguity with improved long-term success have been previously demonstrated $[19,20]$. It will be important to continue observing real-world clinical outcomes as more data become available from standardized AI protocols.

\subsection{Limitations}

The primary limitations of this study are consequences of the non-randomized retrospective design from a single site. In particular, the results may lack generalizability due to sitespecific factors such as procedural workflow, level of operator experience and skill, or unique characteristics of the patient population. Procedure-related complications were only systematically and prospectively collected since 2015 , such that subclinical complications occurring prior to 2015 may have been missed. Furthermore, there is potential that unmeasured variables related to either the patients or procedures could confound the presented results. Finally, as we did not formally compare our approach to other workflows, we cannot offer proof that this particular workflow has superior characteristics in terms of procedural time or variability.

\section{Conclusion}

A highly standardized workflow for AF ablation greatly reduced procedural variability by using $3 \mathrm{D}$ rotational angiography and image integration for the mapping phase, and VisitagAI guided ablation enabled by the ST CF-sensing catheter for the ablation phase. This workflow led to predictably low procedure and fluoroscopy times, with good clinical outcomes as seen in the high rates of freedom from atrial arrhythmia recurrence in both PAF and PsAF populations.

Acknowledgments The authors appreciate the input of Megan Estes, Michael Fisher, and Amanda Coleman for their assistance in the study and manuscript development process.

Funding information This work was supported by Biosense Webster, Inc.

\section{Compliance with ethical standards}

Conflict of interest T De Potter, S Chatzikyriakou, E Silva Garcia, and P Geelen have no disclosures. T Strisciuglio has been supported by a research grant provided by the CardioPath $\mathrm{PhD}$ program. TD Hunter is an employee of CTI Clinical Trial and Consulting Services, which is a consultant to Biosense Webster, Inc. L Boo is an employee of Biosense Webster, Inc.

Open Access This article is distributed under the terms of the Creative Commons Attribution 4.0 International License (http:// creativecommons.org/licenses/by/4.0/), which permits unrestricted use, distribution, and reproduction in any medium, provided you give appropriate credit to the original author(s) and the source, provide a link to the Creative Commons license, and indicate if changes were made.

\section{References}

1. Afzal MR, Chatta J, Samanta A, Waheed S, Mahmoudi M, Vukas $\mathrm{R}$, et al. Use of contact force sensing technology during 
radiofrequency ablation reduces recurrence of atrial fibrillation: a systematic review and meta-analysis. Heart Rhythm. 2015;12(9): 1990-6. https://doi.org/10.1016/j.hrthm.2015.06.026.

2. Das M, Loveday JJ, Wynn GJ, Gomes S, Saeed Y, Bonnett LJ, et al. Ablation index, a novel marker of ablation lesion quality: prediction of pulmonary vein reconnection at repeat electrophysiology study and regional differences in target values. Europace. 2017;19(5): 775-83. https://doi.org/10.1093/europace/euw105.

3. Phlips T, Taghji P, El Haddad M, Wolf M, Knecht S, Vandekerckhove Y, et al. Improving procedural and one-year outcome after contact force-guided pulmonary vein isolation: the role of interlesion distance, ablation index, and contact force variability in the 'CLOSE'-protocol. Europace. 2018;20:f419-27. https://doi. org/10.1093/europace/eux376.

4. De Potter T Jr, Bardhaj G, Viggiano A, Morrice K, Geelen P. Threedimensional rotational angiography as a Periprocedural imaging tool in atrial fibrillation ablation. Arrhythm Electrophysiol Rev. 2014;3(3):173-6. https://doi.org/10.15420/aer.2014.3.3.173.

5. Natale A, Reddy VY, Monir G, Wilber DJ, Lindsay BD, McElderry HT, et al. Paroxysmal AF catheter ablation with a contact force sensing catheter: Results of the prospective, multicenter SMARTAF trial. J Am Coll Cardiol. 2014;64(7):647-56. https://doi.org/10. 1016/j.jacc.2014.04.072.

6. Hussein AA, Barakat AF, Saliba WI, Tarakji KG, Bassiouny M, Baranowski B, et al. Persistent atrial fibrillation ablation with or without contact force sensing. J Cardiovasc Electrophysiol. 2017;28(5):483-8. https://doi.org/10.1111/jce.13179.

7. Tanaka N, Inoue K, Tanaka K, Toyoshima Y, Oka T, Okada M, et al. Automated ablation annotation algorithm reduces re-conduction of isolated pulmonary vein and improves outcome after catheter ablation for atrial fibrillation. Circ J. 2017;81(11):1596-602. https://doi. org/10.1253/circj.CJ-17-0195.

8. Goldsweig AM, Abbott JD, Aronow HD. Physician and patient radiation exposure during endovascular procedures. Curr Treat Options Cardiovasc Med. 2017;19(2):10. https://doi.org/10.1007/ s11936-017-0507-9.

9. Berg BP, Denton BT, Ayca Erdogan S, Rohleder T, Huschka T. Optimal booking and scheduling in outpatient procedure centers. Comput Oper Res. 2014;50:24-37. https://doi.org/10.1016/j.cor.2014.04.007.

10. Baher A, Kheirkhahan M, Rechenmacher SJ, Marashly Q, Kholmovski EG, Siebermair J, et al. High-power radiofrequency catheter ablation of atrial fibrillation: Using Late Gadolinium Enhancement Magnetic Resonance Imaging as a Novel Index of Esophageal Injury. JACC Clin Electrophysiol. 2018;4(12):158394. https://doi.org/10.1016/j.jacep.2018.07.017.

11. Barkagan M, Contreras-Valdes FM, Leshem E, Buxton AE, Nakagawa H, Anter E. High-power and short-duration ablation for pulmonary vein isolation: safety, efficacy, and long-term durability. J Cardiovasc Electrophysiol. 2018;29(9):1287-96. https:// doi.org/10.1111/jce.13651.
12. Bourier F, Duchateau J, Vlachos K, Lam A, Martin CA, Takigawa $\mathrm{M}$, et al. High-power short-duration versus standard radiofrequency ablation: insights on lesion metrics. J Cardiovasc Electrophysiol. 2018;29(11):1570-5. https://doi.org/10.1111/jce.13724.

13. Leshem E, Zilberman I, Tschabrunn CM, Barkagan M, ContrerasValdes FM, Govari A, et al. High-power and short-duration ablation for pulmonary vein isolation: Biophysical Characterization. JACC Clin Electrophysiol. 2018;4(4):467-79. https://doi.org/10.1016/j. jacep.2017.11.018.

14. Winkle RA, Mead RH, Engel G, Patrawala RA. Atrial fibrillation ablation: "perpetual motion" of open irrigated tip catheters at $50 \mathrm{~W}$ is safe and improves outcomes. Pacing Clin Electrophysiol. 2011;34(5): 531-9. https://doi.org/10.1111/j.1540-8159.2010.02990.x.

15. Winkle RA, Moskovitz R, Hardwin Mead R, Engel G, Kong MH, Fleming $\mathrm{W}$, et al. Atrial fibrillation ablation using very short duration $50 \mathrm{~W}$ ablations and contact force sensing catheters. J Interv Card Electrophysiol. 2018;52(1):1-8. https://doi.org/10.1007/ s10840-018-0322-6.

16. Wakili R, Clauss S, Schmidt V, Ulbrich M, Hahnefeld A, Schussler F, et al. Impact of real-time contact force and impedance measurement in pulmonary vein isolation procedures for treatment of atrial fibrillation. Clin Res Cardiol. 2014;103(2):97-106. https://doi.org/ 10.1007/s00392-013-0625-7.

17. Ciconte G, Baltogiannis G, de Asmundis C, Sieira J, Conte G, Di Giovanni G, et al. Circumferential pulmonary vein isolation as index procedure for persistent atrial fibrillation: a comparison between radiofrequency catheter ablation and second-generation cryoballoon ablation. Europace. 2015;17(4):559-65. https://doi. org/10.1093/europace/euu350.

18. Taghji P, El Haddad M, Phlips T, Wolf M, Knecht S, Vandekerckhove $\mathrm{Y}$, et al. Evaluation of a strategy aiming to enclose the pulmonary veins with contiguous and optimized radiofrequency lesions in paroxysmal atrial fibrillation: A pilot study. JACC Clin Electrophysiol. 2018;4(1):99-108. https://doi.org/10.1016/j. jacep.2017.06.023.

19. Zucchelli G, Sirico G, Rebellato L, Marini M, Stabile G, Del Greco $\mathrm{M}$, et al. Contiguity between ablation lesions and strict catheter stability settings assessed by VISITAG(TM) module improve clinical outcomes of paroxysmal atrial fibrillation ablation - results from the VISITALY study. Circ J. 2018;82(4):974-82. https://doi. org/10.1253/circj.CJ-17-0421.

20. Reddy VY, Pollak S, Lindsay BD, McElderry HT, Natale A, Kantipudi C, et al. Relationship between catheter stability and 12month success after pulmonary vein isolation: A subanalysis of the SMART-AF trial. JACC Clin Electrophysiol. 2016;2(6):691-9. https://doi.org/10.1016/j.jacep.2016.07.014.

Publisher's note Springer Nature remains neutral with regard to jurisdictional claims in published maps and institutional affiliations. 